\title{
AN AIDED NAVIGATION POST PROCESSING FILTER FOR DETAILED SEABED MAPPING UUVS
}

\author{
Kenneth Gade and Bjørn Jalving \\ Norwegian Defence Research Establishment (FFI) \\ P. O. Box 25, 2007 Kjeller, Norway
}

\begin{abstract}
HUGIN is an untethered underwater vehicle (UUV) intended for bathymetric data collection for detailed seabed surveying. The HUGIN sensor suite, consisting of standard commercially available navigation sensors and a multibeam echosounder, is briefly presented. A Kalman filter based post processing integration of UUV sensors and survey vessel sensors is discussed. Resulting UUV position and heading accuracy and important characteristics of the post processing filter is shown with simulation results and results from a commercial survey operation. Finally, we briefly show how the claimed position and heading accuracy has been verified.
\end{abstract}

\section{Introduction}

In the HUGIN development program two untethered underwater vehicles have been produced. The vehicles are fitted with a Kongsberg Simrad EM 3000 multibeam echosounder for underwater surveys to depths of $600 \mathrm{~m}$. HUGIN I had its first sea trial in summer 1996 and has been used as a test and demonstration platform. HUGIN II was in spring 1998 put into commercial operation, offering services to the survey market. The HUGIN development program is a co-operation between Norwegian Defence Research Establishment (FFI), Kongsberg Simrad AS, Norwegian Underwater Intervention AS (NUI) and Statoil, Størkersen et. al. [1].

The aided post processing navigation system presented in this paper, was used in a commercial survey operation (Åsgard Transport) with HUGIN I on the Norwegian continental shelf in autumn 1997. The claimed positioning accuracy has been verified and documented in Jalving \& Gade [2]. The aided navigation system is currently being integrated in the Neptune/Merlin commercial post processing package from Kongsberg Simrad AS.

\section{UUV positioning}

The objective of the HUGIN system is to collect data for detailed seabed mapping. Fig. 1 shows the navigation systems and sensors necessary for positioning of multibeam echosounder data in global coordinates. A commercial survey vessel will typically have its position provided by Differential Global Positioning System (DGPS). The position of the HUGIN vehicle relative to the surface vessel is measured by means of the High Precision Acoustic Positioning system
(HiPAP) from Kongsberg Simrad AS. In order to determine the orientation of the EM 3000 transducer, which is necessary for positioning of the EM 3000 footprint relative to the UUV, HUGIN is equipped with a Seatex Motion Reference Unit (MRU), which among several data, outputs the vehicle's roll and pitch angle. MRU has an inertial sensor assembly of three gyros and three accelerometers. Heading is measured by a Leica Digital Magnetic Compass and depth is measured with a Digiquartz $9001 \mathrm{~K}-101$ pressure transmitter. An EDO 3050 Doppler Velocity Log (DVL) provides a velocity measurement.

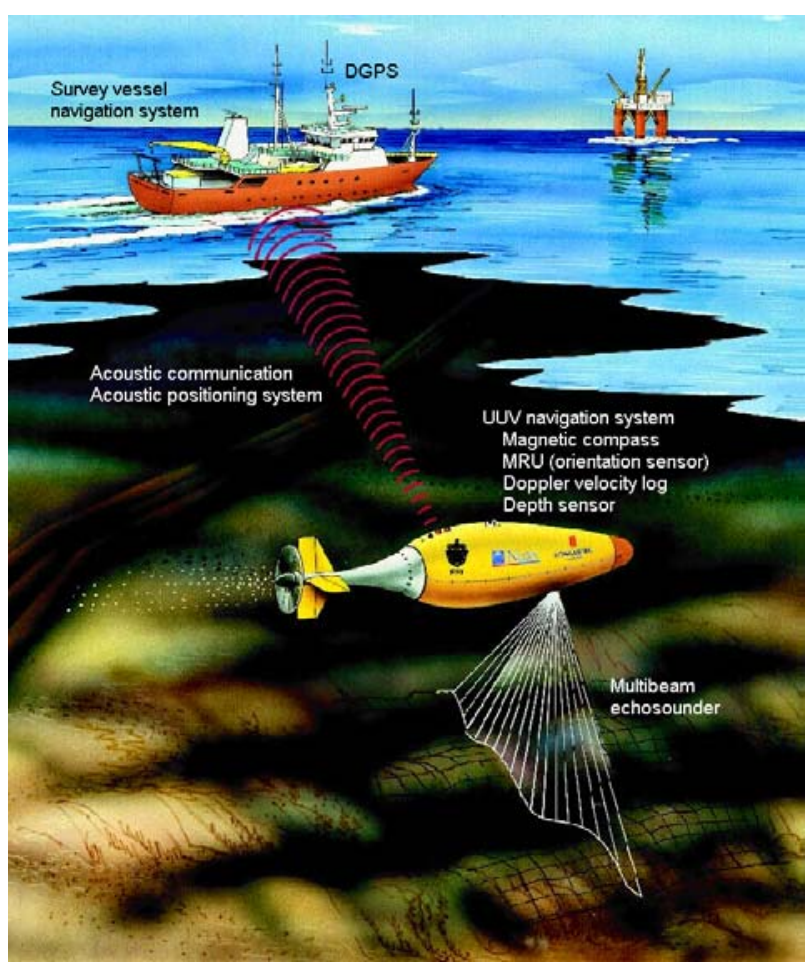

Fig. 1. A seabed mapping scenario with the HUGIN system.

During a survey mission, EM 3000 multibeam echosounder data and HUGIN sensor data are stored locally in the UUV on a hard disk. After a mission, these data are merged with 
DGPS/HiPAP position data stored aboard the survey vessel, in the post processing filter described below.

From a complete EM 3000 footprint positioning error budget presented in Jalving \& Gade [3], it is seen that the horizontal UUV position measurement (combined DGPS and HiPAP) and the UUV magnetic heading measurement are candidates for substantially improved accuracy.

\section{Kalman filter design}

Estimating horizontal position and heading, a possible basis includes:

\section{- Sensor measurements}

- System knowledge (i.e. models of the UUV, its sensors and the environment)

- Control variables (i.e. rudder deflection, stern plane deflection and propeller revolution)

By combining measured control variables with a hydrodynamic UUV model and a sea current model, it is possible to calculate estimates of for instance linear and angular velocity. However, due to considerable model uncertainty, these estimates are far less accurate than the measurements from the Doppler velocity log and the MRU gyros, and thus this strategy offers no significant aid to the estimates.

Consequently, the position and heading estimates should be based on sensor measurements and knowledge of their error models. The optimal way to combine this information is by means of a Kalman filter. Since we have measurements of the wanted quantities (position and heading), it is convenient to use an error-state Kalman filter. Rather than estimating the position and heading directly, this filter estimates errors in measured and computed quantities.

In order to estimate any errors, we need some kind of redundant information, which in case of an error-state Kalman filter should be realized by providing more than one measurement of each state. As seen in Table 1, no such measurements are available, and hence we need external computations, i.e. some combination of measurements calculating the desired quantity:

- An alternative position can be calculated by integrating the body fixed velocity vector in the direction given by the measured roll, pitch and heading (dead reckoning).

- Integration of the angular rates with roll and pitch can give an alternative heading (compensating for earth's angular rate).

In this manner we get two independent positions and headings available. The independent positions and headings also have complementary characteristics. Whereas the measured quantities may have significant high-frequency errors, the computed quantities will be very accurate in the highfrequency band, as they are based on measurements of the derivative. On the other hand the computed quantities have very poor low-frequency properties, drifting off the true value due to sensor errors. Hence, the limited errors of the measured position and heading are vital to ensure lowfrequency stability of the Kalman filter estimates. Altogether a combined solution offers increased low and high frequency accuracy.

Table 1. Available sensor measurements for the integrated navigation system

\begin{tabular}{|c|c|c|}
\hline Sensor & Measurement & $\begin{array}{l}\text { Typical accu- } \\
\text { racy }(1 \sigma)\end{array}$ \\
\hline $\begin{array}{l}\text { HiPAP + } \\
\text { DGPS }\end{array}$ & $\begin{array}{l}\text { UUV position (relative } \\
\text { earth) }\end{array}$ & $2 m-4 m$ \\
\hline MRU & $\begin{array}{l}\text { Roll and pitch } \\
\text { UUV angular velocity } \\
\text { (relative the inertial frame) } \\
\text { projected into the body } \\
\text { coordinate system }\end{array}$ & $\begin{array}{l}0.07^{\circ} \\
>10^{\circ} / \mathrm{h}\end{array}$ \\
\hline Compass & Heading & $2^{\circ}-3^{\circ}$ \\
\hline DVL & $\begin{array}{l}\text { UUV velocity (relative the } \\
\text { seabed) projected into the } \\
\text { body coordinate system }\end{array}$ & $0.015 \mathrm{~m} / \mathrm{s}$ \\
\hline $\begin{array}{l}\text { Pressure } \\
\text { sensor }\end{array}$ & Depth (after calculations) & $0.1 \mathrm{~m}$ \\
\hline
\end{tabular}

Measurements to the error-state Kalman filter are the difference between measured and computed quantities, as shown in Fig. 2.

Based on the measurements and sensor error models, the Kalman filter estimates all the colored sensor errors and the errors in the computed quantities. 


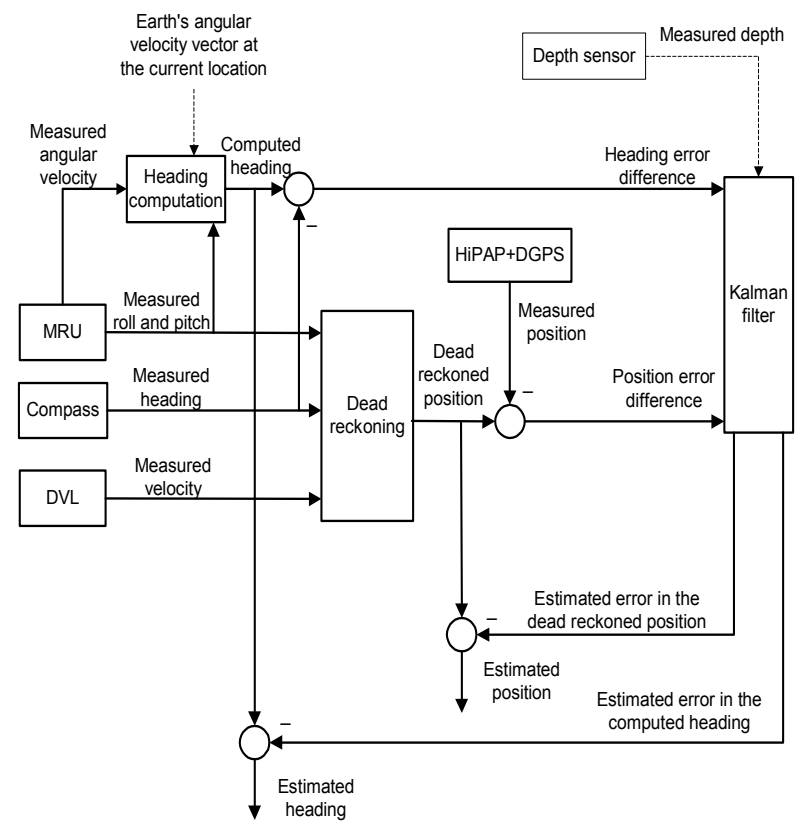

Fig. 2. Kalman filter structure

The sensor error models were found by established system identification methods. Sensor data from both sea trials and static conditions (fixed HiPAP transponder, fixed DGPS receiver and fixed UUV orientation) was used. The errors were modelled as combinations of white and colored noise as shown in Table 2. The colored parts are well represented by first order Markov processes.

The colored sensor errors thus sum up to four Kalman filter states (the position measurement error has both a north and east component). Further, one single integration gives a new state, leading to three states from the estimation of errors in the computed quantities. Thus, the Kalman filter has a total of seven states.

Table 2. Summary of the sensor error models

\begin{tabular}{|l|c|c|}
\hline Sensor & Colored part & White-noise part \\
\hline HiPAP + DGPS & $\mathbf{X}$ & $\mathbf{X}$ \\
MRU, roll and pitch & $\mathbf{X}$ \\
MRU, angular rate & $\mathbf{X}$ & \\
Compass & $\mathbf{X}$ & $\mathbf{X}$ \\
DVL & & $\mathbf{X}$ \\
\hline
\end{tabular}

The final position and heading estimates can be calculated by subtracting the corresponding error estimates from either the measured or the computed quantities. As shown in Fig. 2 , the latter is preferred, motivated by the following:

- White-noise is not possible to estimate, hence only the colored parts of the errors in the position and heading measurements are estimated. Consequently, a measurement based estimate would contain white-noise. As for the computed quantities, the integration process has eliminated the white noise component from the MRU, compass and Doppler velocity log, and the entire error may be estimated.

- The computed quantities are higher order processes, and are much more correlated in time than the first order Markov processes. Hence, errors in the dead reckoned position and the computed heading are far more predictable, giving more accurate a priori estimates and thus reduced a posteriori estimation uncertainty. During measurement drop-outs, the Kalman filter can only predict the errors, and the predictability is particularly important.

- Due to occasional measurement drop-outs of the DGPS or HiPAP, the more reliable dead reckoned position is a preferred basis.

\section{Smoothing}

A Kalman filter is recursive and its estimates at time $t_{k}$ are based on all measurements prior to and including $t_{k}$. Since there is no real-time requirement in the post processing, measurements after $t_{k}$ should also be utilized. The matter of finding an optimal estimate based on both previous and future measurements is referred to as smoothing.

Smoothing has several advantages compared to just a conventional Kalman filter:

- Since all measurements are known a priori, there is no delay in the estimates.

- The smoothed estimates are in accordance with the process model. This is different from a conventional Kalman filter, where the process model is used only in the prediction part. Thus, when updating the filter, unexpected measurements lead to steps in the a posteriori estimate.

- In a conventional Kalman filter, estimating the current state, most weight is put on the latest measurements (due to the states' correlation in time). Thus making smoothed estimates, the number of relevant measurements is doubled. 
- During measurement drop-outs, the estimation uncertainty of an ordinary Kalman filter increases in accordance with the process noise until the measurement is back. Knowledge of the next measurement reduces the uncertainty increase-rate of the smoothed estimate and causes its maximum to occur in the middle of the dropout time interval.

To find the smoothed estimates, first the ordinary Kalman filter is run through the whole time series, saving all estimates and covariance matrices. The saved data is then processed recursively backwards in time using an optimal smoothing algorithm (Minkler \& Minkler [4] or Gelb [5]) adjusting the filtered estimates.

\section{Filter characteristics and performance}

This section includes results from tests where both simulated measurements and measurements from sea trials were applied to the designed filter

\section{A. Observability}

All the Kalman filter states are observable. However, errors in the position measurement that are more low-frequent than the drift in the dead reckoned position, are not possible to estimate. In the compass measurement though, both highfrequency and low-frequency errors are estimated (assuming UUV velocity not zero). The high-frequency error is found by means of the gyros (computed heading), and the lowfrequency part is estimated by observing the drift in the dead reckoned position (with the aid of the DGPS/HiPAP position measurement).

\section{B. Simulation results}

Simulations are very useful for demonstrating typical filter characteristics. A set of simulated measurements was derived from dynamical models of the UUV, the environment and sensor errors. The subset of sensor error models which was also implemented in the Kalman filter, was based on the same error-modelling. The simulated UUV trajectory is shown in Fig. 3, the UUV forward velocity was $2.1 \mathrm{~m} / \mathrm{s}$.
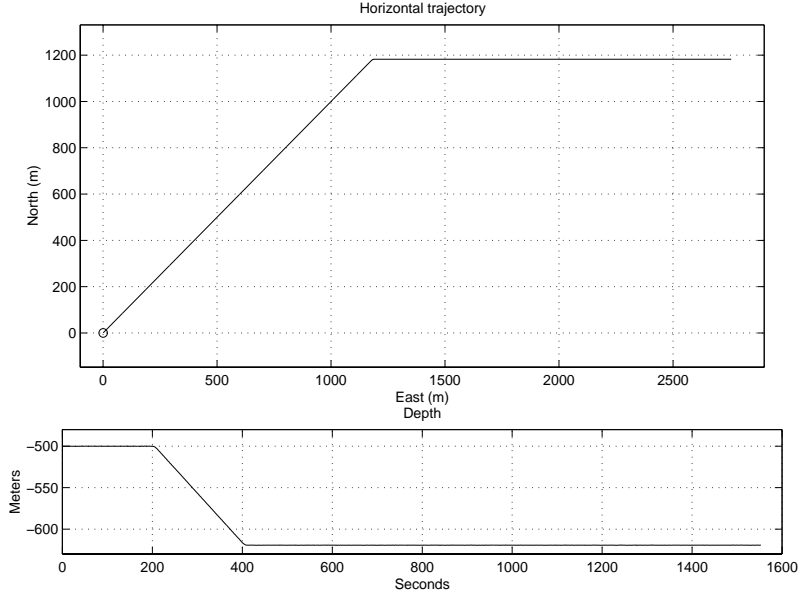

Fig. 3. Simulated UUV trajectory. Circle: starting point.

\section{1) Position estimation}

Fig. 4 shows the results from the position estimation. The UUV has moved straight eastwards, and clearly the position measurement contains both high- and low-frequency noise. The dead reckoned position is drifting, but is very smooth, which in this case means small high-frequency errors. As the Kalman filtered estimate is susceptible to measurement noise, it is not as smooth as the dead-reckoned position. However, the smoothed estimate is both smooth and very close to the truth.

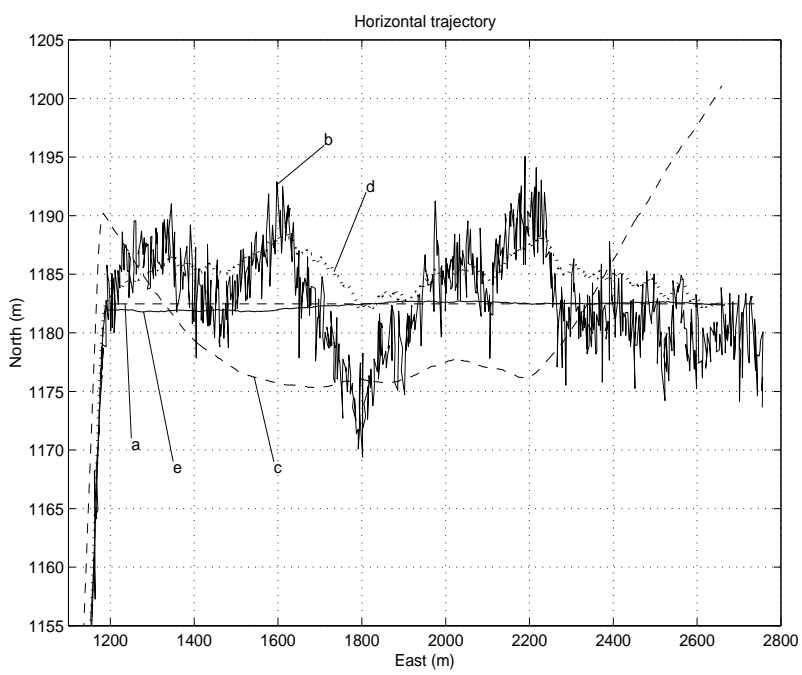

Fig. 4. Horizontal UUV trajectory. True (a), measured (b), deadreckoned (c), filtered estimate (d), smoothed estimate (e)

The accuracy of the north and east position estimates is shown in Fig. 5. For comparison, the position measurement uncertainty is also indicated. The change in this quantity is due to the depth increase, leading to lower HiPAP accuracy. 

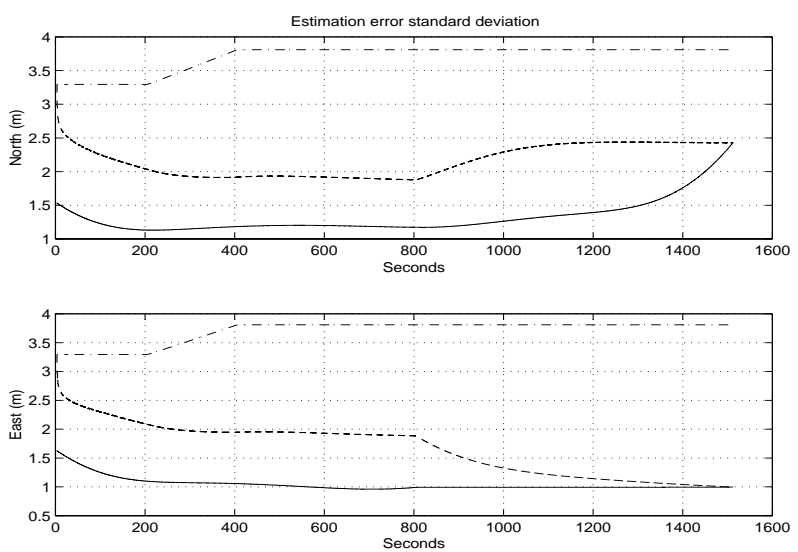

Fig. 5. Position estimation uncertainty (standard deviation). Filtered estimate (dashed), smoothed estimate (solid), measurement uncertainty (dash-dot)

Because the filter is initialized by the position measurement, the accuracy of the filtered estimate equals the measurement accuracy in the first time step. As the number of relevant measurements increases, the accuracy converges to below 2 meters. At time $=800$ seconds, the UUV starts a $45^{\circ}$ turn, leading to increased uncertainty in the north direction and decreased uncertainty in the east direction. This demonstrates the difference in accuracy along-track and crosstrack, which is due to a similar characteristic of the deadreckon drift. The main contributor to the dead-reckon drift is the compass-error, whose drift contribution is of first order in the cross-track direction, but only of second order alongtrack.

From the figure it is evident that the smoothed estimate is generally better, but at the last time step there are no more future measurements available, and the smoothed estimate equals the filtered, both in accuracy and value.

\section{2) Heading estimation}

Fig. 6 shows the heading estimation. The graphs are very similar to the corresponding graphs of the position estimation. In addition it is apparent that both the computed heading and the filtered heading estimate are initialized by the compass measurement.

The heading estimation error standard deviation is shown in Fig. 7. The smoothed solution offers more than a tenfold improvement in accuracy over the compass measurement.
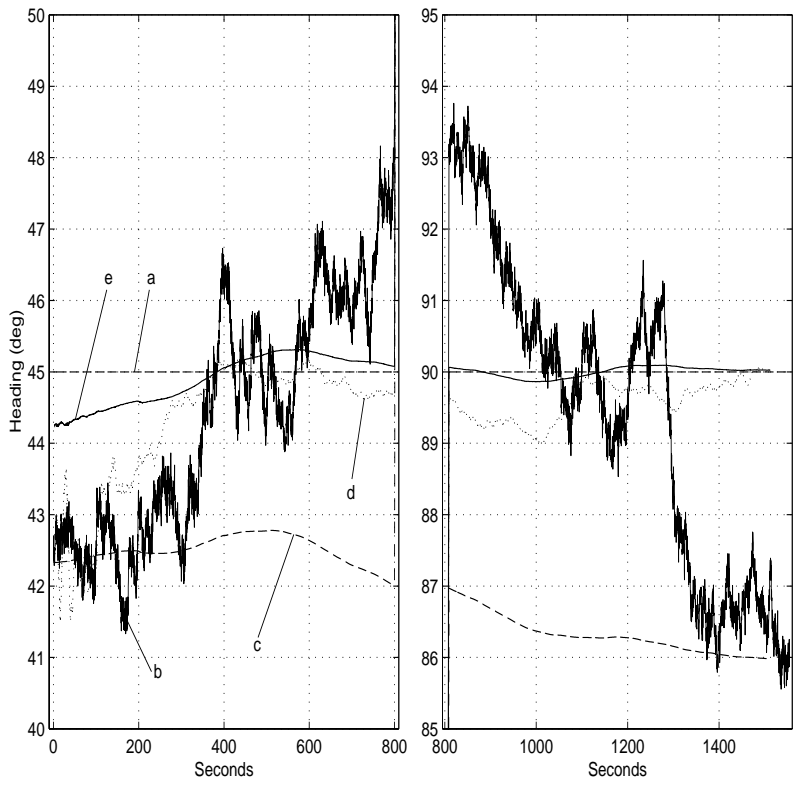

Fig. 6. UUV heading. True (a), measured (b), computed (c), filtered estimate (d), smoothed estimate (e)

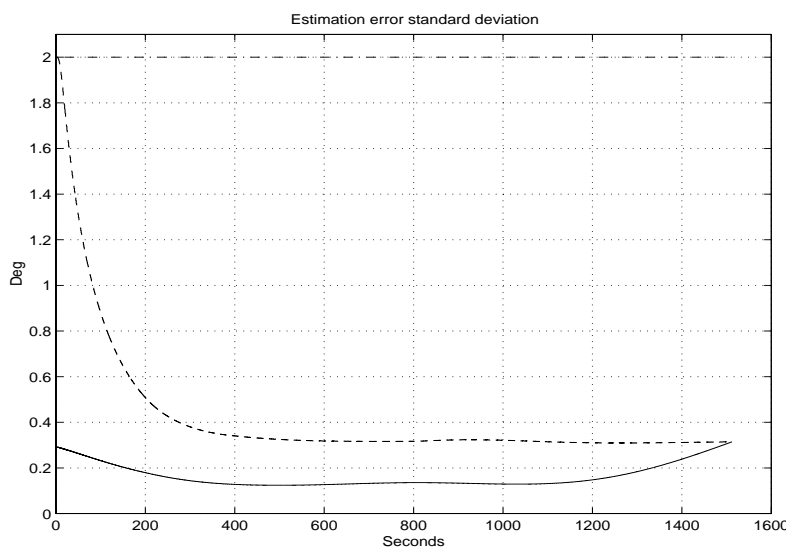

Fig. 7. Heading estimation uncertainty (standard deviation). Filtered estimate (dashed), smoothed estimate (solid), measurement uncertainty (dash-dot)

\section{Results from real surveys}

Fig. 8 shows the horizontal trajectory from a real survey in Boknafjorden in Norway, at a depth of approximately 320 $\mathrm{m}$. The Kalman filtered position estimate is clearly more susceptible to measurement errors than the smoothed. The considerable drift in the dead-reckoned position is due to a significant steady compass error. 


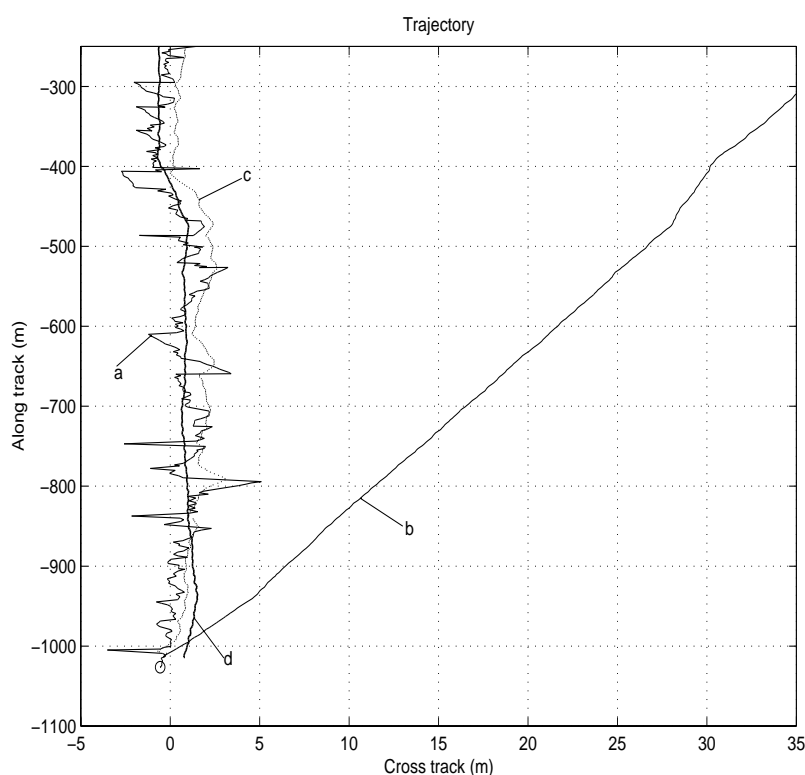

Fig. 8. Horizontal UUV trajectory. Measured (a), dead-reckoned (b), filtered estimate (c), smoothed estimate (d)

In Fig. 9 measured and estimated heading are shown. According to the filter there is a compass error in the order of $3^{\circ}$. At the time of this survey, declination and the UUV's magnetic signature was not yet compensated for.

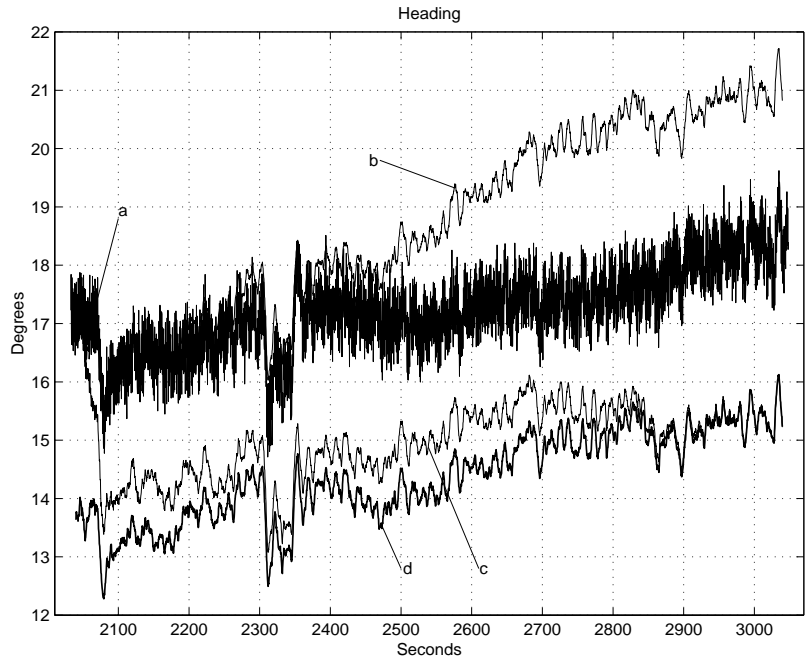

Fig. 9. UUV heading. Measured (a), computed (b), filtered estimate (c), smoothed estimate (d)

\section{Verification of performance}

There are several established methods for determining the quality of the produced Digital Terrain Model (DTM). An obvious method would be to map a marker placed on the seabed in a known location. At present, no such data is available, but natural features, for instance rocks, are visible on the sonar data and can be classified as objects. In cases where we have overlapping sonar data and can identify the same object on two footprints, an offset between the two observations indicates DTM position error(s). According to Jalving and Gade [3], the UUV heading and position uncertainties are the main contributors to the DTM position uncertainty. Comparing the offset between the observations prior to ${ }^{1}$ and after the filtering thus gives an idea of the improvement achieved in the post processing.

The observed offsets can also be compared with theoretical values calculated from the uncertainty in the position and heading. Prior to the filtering, the position and heading uncertainties are given by the DGPS/HiPAP and compass accuracies (listed in Table 1). For the observations in the filtered data set, the theoretical value is based on the Kalman filter standard deviation of the position and heading error estimate. Due to a temporarily invidious installation of a magnetic valve and a few other non-ideal circumstances, we assumed a heading uncertainty of $0.8^{\circ}$ instead of the much better Kalman filter standard deviation. Table 3 summarizes comparisons for all the objects we found in the runs from Åsgard Transport and Boknafjorden. Each object is mapped two times separated with a time interval of 30 minutes or more, passing the object from opposite directions. Most of the objects from the Åsgard Transport were at a depth about 350 meters.

For some objects there was no measurable position offset between the two observations, which is indicated in Table 3 by using the "less than" sign $(<)$. The value after the sign is dependent upon the accuracy of the observation. When computing average, this value is divided by two.

In Table 3 we notice a significant improvement in the filtered data. Furthermore, we can compare the observation position offset after filtering with its theoretical standard deviation. Assuming normally distributed errors, $68 \%$ of the observed position offsets should be within its standard deviation. The bold figures indicate an offset exceeding the standard deviation, and we have 19 of 28 inside, which is exactly $68 \%$ ! However, this test only compares each value with a boundary, not taking into account how far from the boundary they are. Investigating the average actually indicates a better performance than anticipated. This may suggest that the filtered heading uncertainty of $0.8^{\circ}$ used in the theoretical standard deviation calculations is too conservative. In Table 3 we also notice that the observation position

\footnotetext{
${ }^{1}$ Prior to the filtering, the measured position and heading
} are used directly. 
offsets prior to filtering are slightly better than the theoretical values. This is probably due to a counteractive effect of the UUV static magnetic signature.

\section{Conclusions}

The accuracy of seabed maps based on UUV data can be considerably improved by an aided navigation post processing filter.

It has been demonstrated that a combination of all relevant sensors in an error state Kalman filter offers a far more accurate position and heading, than direct use of the position and heading measurements. Further, the Kalman filtered estimates may be considerably enhanced through a smoothing algorithm. At $300 \mathrm{~m}$ depth, a UUV position accuracy of $1 \mathrm{~m}$ $(1 \sigma)$ and a heading accuracy of $0.5^{\circ}(1 \sigma)$ has been achieved.
Table 3 Comparison of object observation position offset in filtered data set (smoothed position and heading) and unfiltered data set (combined DGPS/HiPAP position and compass heading). Theoretical uncertainties of the two data sets are also calculated.

\begin{tabular}{|c|c|c|c|c|c|c|c|c|}
\hline \multirow[b]{2}{*}{$\begin{array}{c}\text { Object } \\
\text { no. }\end{array}$} & \multicolumn{2}{|c|}{$\begin{array}{l}\text { Observation } \\
\text { position offset } \\
\text { prior to filtering } \\
\text { (m) }\end{array}$} & \multicolumn{2}{|c|}{$\begin{array}{c}\text { Observation } \\
\text { position offset } \\
\text { after smoothing } \\
\text { (m) }\end{array}$} & \multicolumn{2}{|c|}{$\begin{array}{c}\text { Theoretical } \\
\text { uncertainty prior } \\
\text { to filtering }(1 \sigma) \\
(\mathrm{m})\end{array}$} & \multicolumn{2}{|c|}{$\begin{array}{c}\text { Theoretical } \\
\text { uncertainty after } \\
\text { smoothing }(1 \sigma) \\
(\mathrm{m})\end{array}$} \\
\hline & North & East & North & East & North & East & North & East \\
\hline 1 & 7.5 & 2.0 & $<0.5$ & $<0.5$ & 5.12 & 3.40 & 1.17 & 1.00 \\
\hline 2 & 4.6 & 2.3 & $<0.5$ & $<0.5$ & 5.06 & 3.40 & 1.16 & 1.00 \\
\hline 3 & 1.8 & 4.5 & $<0.5$ & 0.8 & 4.92 & 3.39 & 1.15 & 1.00 \\
\hline 4 & 3.0 & 1.2 & $<0.5$ & 2.0 & 4.92 & 3.38 & 1.15 & 1.00 \\
\hline 5 & 6.2 & 2.0 & $<0.5$ & $<0.5$ & 5.43 & 3.42 & 1.21 & 1.00 \\
\hline 6 & 4.2 & 1.3 & 1.6 & $<0.5$ & 5.59 & 3.41 & 1.22 & 1.00 \\
\hline 7 & 7.0 & 5.0 & 1.5 & 1.2 & 5.48 & 3.32 & 1.21 & 0.99 \\
\hline 8 & 8.0 & $<0.5$ & 1.7 & 0.6 & 4.64 & 3.32 & 1.12 & 0.99 \\
\hline 9 & 8.5 & 0.7 & 2.0 & 0.5 & 4.26 & 3.32 & 1.08 & 0.99 \\
\hline 10 & 3.6 & 2.2 & 1.7 & 0.5 & 4.82 & 3.31 & 1.14 & 0.99 \\
\hline 11 & 0.7 & 2.0 & 1.3 & 2.0 & 3.28 & 3.24 & 1.13 & 1.27 \\
\hline 12 & 2.9 & 1.1 & 0.3 & $<0.2$ & 3.24 & 3.24 & 1.13 & 1.27 \\
\hline 13 & 0.1 & 0.8 & 0.1 & 0.3 & 3.30 & 3.24 & 1.14 & 1.27 \\
\hline 14 & 3.3 & 2.3 & $<0.5$ & $<0.5$ & 4.17 & 3.76 & 0.84 & 0.79 \\
\hline Average: & 4.39 & 1.98 & 0.84 & 0.66 & 4.59 & 3.37 & 1.13 & 1.04 \\
\hline
\end{tabular}

\section{References}

[1] N. Størkersen, J. Kristensen, A. Indreeide, J. Seim and T. Glancy, "HUGIN - UUV for seabed surveying", Sea Technology, February 1998.

[2] B. Jalving and K. Gade, Positioning Accuracy for Seabed Surveying Untethered Underwater Vehicles (UUVs), submitted to IEEE Journal of Oceanic Engineering.

[3] B. Jalving and K. Gade, Positioning Accuracy for the HUGIN Detailed Seabed Mapping UUV, Proceedings from Oceans '98, 28. Sept. - 1. Oct. 1998, Nice, France

[4] G. Minkler and J. Minkler, Theory and Application of Kalman Filtering, Magellan Book Company, 1993.

[5] A. Gelb, Applied Optimal Estimation, The MIT Press, 1974 